\title{
Subtalar Dislocation: Long-Term Follow-Up and CT-Morphology
}

\author{
Stefanie Hoelscher-Doht ${ }^{1}{ }^{*}$, Sönke P. Frey ${ }^{1}$, Sebastian Kiesel' ${ }^{2}$, Rainer H. Meffert ${ }^{1}$, \\ Hendrik Jansen ${ }^{1}$ \\ ${ }^{1}$ Department of Trauma, Hand, Plastic and Reconstructive Surgery, University Clinics of Wuerzburg, Wuerzburg, \\ Germany \\ ${ }^{2}$ Department of Orthopedics and Trauma Surgery, Caritas-Hospital, Bad Mergentheim, Germany \\ Email: ${ }^{*}$ hoelscher s@klinik.uni-wuerzburg.de
}

Received 3 February 2015; accepted 23 March 2015; published 26 March 2015

Copyright $@ 2015$ by authors and Scientific Research Publishing Inc.

This work is licensed under the Creative Commons Attribution International License (CC BY).

http://creativecommons.org/licenses/by/4.0/

(c) (i) Open Access

\begin{abstract}
Introduction: Although rare, subtalar dislocations are severe injuries with long-term alterations such as clinical dysfunction or painful posttraumatic arthritis. The objective of this study was to investigate long-term morphological changes of subtalar dislocations and to correlate them to clinical function. Based on the conclusions, suggestions for therapy guidelines were made in order to improve the functional outcome. Methods: Twenty-two patients (12 with a medial, 9 with a lateral and 1 with an anterior dislocation) were re-examined with an average follow-up time of 10 years. Radiological results of a computer tomography examination of the hindfoot were related to the clinical outcome, and both were discussed in the context of further parameters, such as additional injuries, time until reduction, and post-reduction treatment. Results: Additional injuries were found to affect the clinical outcome and/or the radiological changes. Predictive factors for limited range of motion were severe skin trauma and traumatic brain injuries. Factors that predicted both poor clinical function and clear signs of arthritis included complex talus, ankle and calcaneus fractures, long time until reduction, and infection. Conclusions: In many cases, radiological results can be correlated to clinical outcomes. However, the modulating effects of additional injuries should be considered when planning therapy. In cases with additional fractures of the talus and the calcaneus, suffering pain may be reduced by an early arthrodesis of the talo-calcanear joint. In cases involving a long stay on an intensive care unit, early functional treatment by passive motion should be discussed in special cases to improve the clinical outcome.
\end{abstract}

\section{Keywords}

Subtalar, Dislocation, Arthritis, Outcome, Long-Term, CT

\footnotetext{
${ }^{*}$ Corresponding author.
} 


\section{Introduction}

Subtalar dislocation involves the talo-calcanear and talo-navicular joints and is usually caused by an indirect high energy torsional force. Falls from a height (30\% - 90\%) or traffic accidents (50\%) are the most common causes [1] [2]. In 85\% of cases, men are affected [3]. It is a rare injury and only occurs in $1 \%$ - $2 \%$ of all dislocations [2] [4] [5]. Subtalar dislocations can be sub-divided into medial, lateral, and anterior or posterior dislocations depending on the position of the calcaneus relative to the talus. In Figure 1, a lateral dislocation is shown. Forces applied to a plantar-flexed foot in inversion will usually result in a medial subtalar dislocation, whereas a plantar-flexed foot in eversion will result in a lateral dislocation [4] [6]. The most frequent subtalar dislocation is the medial dislocation (80\%). Lateral dislocations account for $17 \%$ and anterior or posterior dislocations are rare (3\%) [7] [8]. Ten to forty per cent of these injuries are open with associated fractures of the talus or os naviculare, with a higher infection rate and with a higher risk of avascular necrosis of the talus [9] [10]. Intra-articular fractures increase the risk of a secondary osteoarthritis of the talo-calcanear and talo-navicular joints [11].

After incurring this complex foot trauma, clinical dysfunction and painful osteoarthritis can lead to severe limitations during daily life. Despite the clinical importance, to date only very limited information is available on the relation between clinical dysfunction and radiological alterations. Many previous studies have presented results with only short follow-up times (1.5 - 4 years) and correlated the clinical outcome with the radiological outcome of X-rays of the ankle in anteroposterior and lateral views [12] [14]. However, alterations to the subtalar joint structures that are typically affected after subtalar dislocations are not easy to detect with X-rays alone. Long-term follow-up studies (from 4 - 26 years) are rare [15] [16]. De Palma et al. re-examined 30 patients with closed isolated subtalar dislocations after 5 - 12 years and suggested a correlation between a poor clinical outcome and arthritis of the subtalar joint [15]. In contrast, Garofalo et al. did not find a strong correlation between signs of arthritis of the subtalar joint and poor clinical outcome and, instead, recommended further investigations to examine long-term alterations of the hindfoot CT scans following subtalar dislocations [16].

In this retrospective study, we report our long-term results of subtalar dislocations, which have been treated in our clinic from 1986 to 2007, and discuss the long-term outcome and complications. The extent of arthritis was assessed by analyzing CT scans of the hindfoot. Correlations between clinical outcomes and radiological changes, as well as the influence of additional injuries will be discussed. Based on the study results evidence-based guidelines to help decide in which cases an early secondary arthrodesis of the talo-calcanear joint may be indicated, will be proposed.

\section{Patients and Methods}

Between 1986 and 2007, 34 patients with subtalar dislocations were treated in our department. In our patient cohort, we had 21 cases with a medial closed, 11 with a lateral closed, 1 with a lateral open and 1 with an anterior open dislocation. We were able to re-examine 22 patients after a mean follow-up of 10 years (3 to 22 years). Despite an intensive search, 4 patients could not be located and 3 died for reasons unrelated to their foot injury. Five patients refused a follow-up examination, although some had a subjective good outcome. Out of the re-examined 22 patients, 12 have had a medial closed dislocation, 9 a lateral (1/9 open, 8/9 closed) dislocation and 1 an anterior open subtalar dislocation. The age at the day of trauma was between 17 and 67 years (mean 34 years) with most occurring between 20 to 30 years. The ratio of men to women was 4.5:1. The cause of subtalar dislocation was a traffic accident in 14 cases and a fall from a height in 8 cases.

The clinical outcome was assessed by the Zwipp score [12] [13], including computer tomography of the hindfoot. The extent of arthritis of the talo-calcanear and the talo-navicular joints, deformation of the talus and osteochondral fragments were the main points of interest. The special points for calcaneus fractures (part 34 and 35 of the Zwipp score) were not taken into account. Furthermore, the cause of injury, type of dislocation, additional injuries, time and mode of reduction, and the post-reduction treatment were documented.

\section{Results}

According to common treatment guidelines for subtalar dislocations, a fast reduction and immobilization for 6 weeks should be performed. Closed reduction was possible in 5 of our 12 medial dislocations. Open reduction was required 7 times. In cases of a lateral subtalar dislocation, closed reduction was possible 4 times and an open reduction was done in 5 of the 9 cases. After reduction the foot was immobilized with a short-leg cast for 6 weeks in 16 


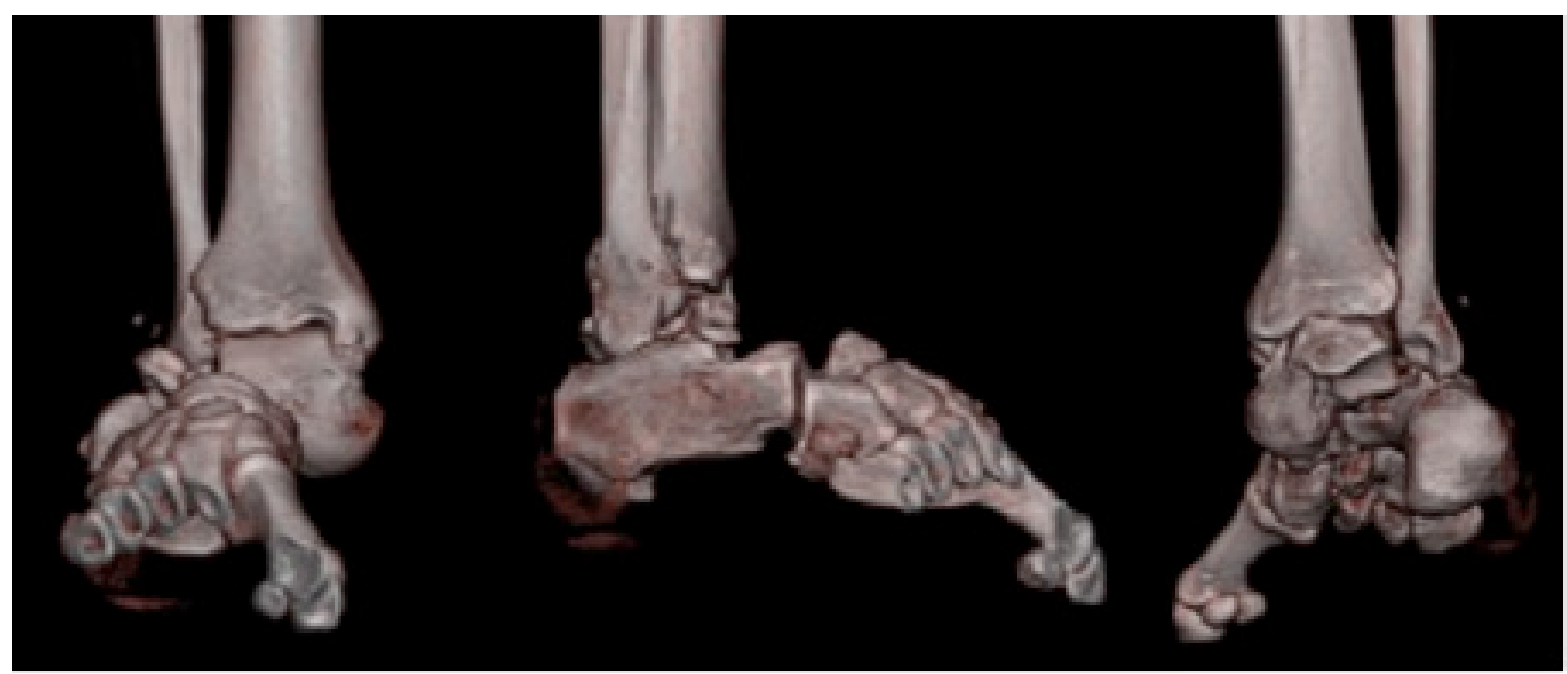

Figure 1. Subtalar dislocations are divided into medial, lateral, and anterior/posterior dislocations depending on the position of the calcaneus in relation to the talus. Here, the foot (and calcaneus) is dislocated lateral of the talus (a lateral subtalar dislocation).

cases. In exceptional cases (skin trauma grade III (Tscherne and Oestern), infection, severe additional fractures) an external fixator for 6 weeks was needed. Fifteen patients had associated fractures of the hind- and/or middle foot. One rare open anterior dislocation was reduced surgically, followed by an external fixator for 6 weeks. Post reduction, necrosis of the talus, a severe possible complication, was not evident in any of the cases.

The mean Zwipp score of all patients was 71.1 (SD \pm 65.8 , maximum $=180$ points) [12] [13]. Based on these Zwipp scores, four possible outcomes were identified: excellent (180 - 135), good (134 - 90), moderate (89 - 45) and poor $(<44)$. Four patients had excellent results and subjectively reported a very good outcome, without experiencing any limitations during everyday life. Their range of motion was normal for the talo-calcanear and talo-navicular joints and no hindfoot deformations were evident. In the computer tomography, no necrosis, osteochondral fragments or even mild sclerosis were detected (Figure 2(A)). In this excellent patient group, three patients had no further fractures of the foot. One patient with associated fractures of the middle foot had a lateral dislocation, which could be closely reduced without delay (within an hour after trauma). Three of the four dislocations could be reduced by a closed procedure and in the one case that required an open reduction, no further complications occurred. The incidence of medial and lateral dislocations was equally distributed. All subtalar dislocations of this group were stable after reduction and could be treated with regular cast immobilization.

Two patients scored between 134 and 90 points (good). They had limitations in their range of motion, especially during supination and inversion, but the dynamic analysis was still scored well. Both patients in this group had medial subtalar dislocations. In one case, instability after reduction persisted and a K-wire transfixation was required. In the other case, only a fracture of the metatarsal $\mathrm{V}$ was found, but this patient had additional thorax and traumatic brain injuries. In this group, the computer tomography showed no deformation of the talus, rare small osteochondral fragments, and only minimal sclerosis.

The third patient group (moderate) was comprised of nine cases (Zwipp score points 89 - 45). The computer tomography revealed moderate alterations, including slight sclerosis and a small reduction in the joint space of the talo-calcanear and talo-navicular joints. Severe signs of osteoarthritis and ossifications were not found (Figure 2(B)). In contrast to the radiological findings, the range of motion was manifestly limited and the dynamic analysis revealed poor results. Clinically, the hindfoot appeared swollen and deformed, but a bony correlate was not found in the computer tomography. In two patients the skin was critical (grade III, Tscherne and Oestern) and they were stabilized with an external fixator instead of cast immobilization. Three patients in this group had a complex talus fracture; in one case the dislocation remained longer than 24 hours and in another case an associated complex ankle fracture had to be treated. Two patients had a traumatic brain injury and required mechanical ventilation in an intensive care unit. The ratio of medial and lateral dislocations was 2:3.

The fourth patient group (poor) had poor Zwipp score $(<45)$. Their range of motion of the foot was manifestly limited (ankle joint: deficit in extension $>10^{\circ}$ and flexion $>20^{\circ}$; talo-calcanear and calcaneo-cuboid joints: defi- 


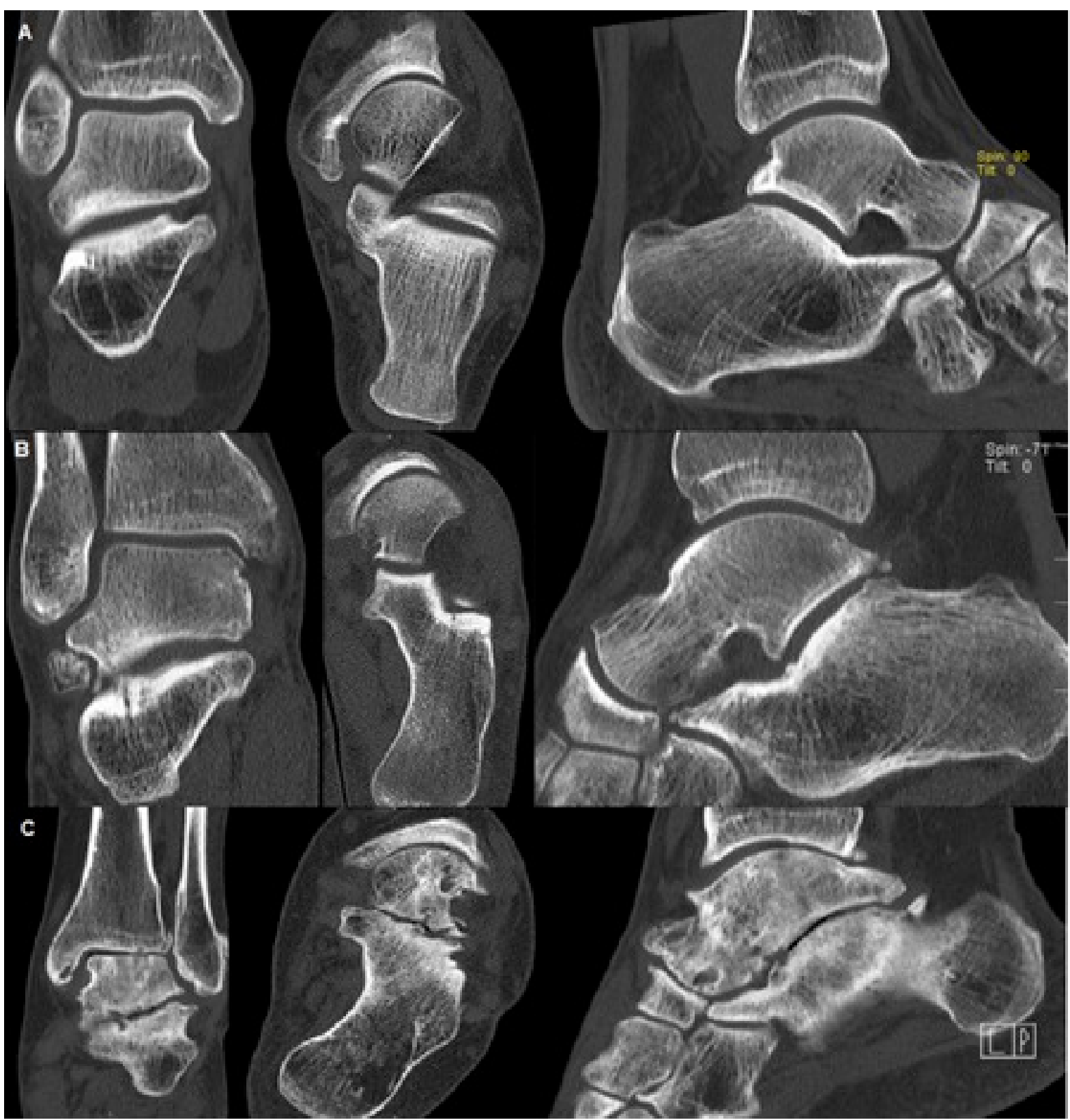

Figure 2. Computer tomography scans of the hindfoot with different extent of arthritis. (A) In the coronal, axial and sagittal view the joint space is intact and no deformation of the talus, osteophyts or subchondral cysts are found (no osteoarthritis); (B) Mild signs of arthritis are found such as a reduction of the joint space, subchondral sclerosis and small osteochondral fragments (coronal, axial and sagittal view); (C) Clear signs of arthritis, including severe reduction of the joint space, subchondral sclerosis and cysts, osteophyts and deformation of the talus are shown in the coronal, axial and sagittal view.

cit in supination $>10^{\circ}$, pronation $>10^{\circ}$, and eversion/inversion $>20^{\circ}$ ). Three patients had complex ankle fractures, fractures of the os naviculare and compound fractures of the calcaneus. In six of the seven cases in this poor patient group, the reduction was done by an open procedure. Four of those patients were stabilized with an external fixator after reduction. The one case, in which the reduction was done by a closed procedure, additional fractures of the calcaneus, os naviculare and ankle were present. The ratio of medial to lateral dislocations was 4:1. In one patient, painful posttraumatic arthritis of the talo-calcanear joint was treated by an arthrodesis of the subtalar joint. In all cases of this poor patient group, there were clear signs of osteoarthritis in the talo-calcanear and talo-navicular joints and also in the ankle joints (Figure 2(C)). Deformation of the talus was moderate in four 
cases. Manifest alteration of the bony hindfoot was found in two cases. In the first case, a medial subtalar dislocation occurred following a fall from a height and an open reduction and transfixation of the talo-calcanear and ankle joints was performed with a Steinmann nail in Thailand (where the injury occurred). Back in Germany, a severe infection of the foot developed. By removing the implant, performing a debridement and attaching an external fixator for 6 weeks, the infection healed successfully under antibiotic therapy. In the second case, an anterior open dislocation was reduced in an open technique and an external fixator was applied for 6 weeks. The computer tomography showed a severe alteration of the talus with a valgus deformation in the coronal view, a reduction of the joint space and multiple osteophytes. The results of the four groups are illustrated in Table 1.

\section{Discussion}

Subtalar dislocations are rare but severe injuries. To reduce the risk of skin necrosis and possible aseptic bone necrosis, an immediate reduction within 6 hours after injury is required to improve the outcome [14] [17] [18]. Medial dislocations can be reduced using a closed procedure. Lateral dislocations are frequently irreducible using a closed procedure because of obstacles like the tibialis posterior tendon, the flexor digitorum longus tendon or the interposition of the talus head in the retinaculum extensorum. In these cases, open reduction is required. Our treatment guidelines, which include an immediate reduction and immobilization for 6 weeks with a short leg cast, correspond to current recommendations [2] [14] [17]-[19]. In cases with persistent instability, we applied a K-wire transfixation of the talo-calcanear and talo-navicular joints. Open dislocations or critical skin situations were treated with an external fixator.

Table 1. Long-term assessment of the outcome after treatment of subtalar dislocations.

\begin{tabular}{|c|c|c|c|c|}
\hline Zwipp score (max 180) & $180-135$ & $134-90$ & $89-45$ & $<45$ \\
\hline Number of patients & 4 & 2 & 9 & 7 \\
\hline \multicolumn{5}{|l|}{ Zwipp score results } \\
\hline Subjective disorders $(+/-15)$ & 13.75 & 4.5 & -1 & -5 \\
\hline Objective clinical signs $(+/-115)$ & 107.5 & 63.5 & 32 & -1 \\
\hline CT scan $(+/-50)$ & 47 & 45 & 35 & 1 \\
\hline \multicolumn{5}{|l|}{ Type of dislocation: } \\
\hline Medial & 2 & 2 & 3 & 5 \\
\hline Lateral & 2 & 0 & 6 & 1 \\
\hline Anterior & 0 & 0 & 0 & 1 \\
\hline Open dislocation & 0 & 0 & 1 & 1 \\
\hline \multicolumn{5}{|l|}{ Treatment: } \\
\hline Reduction by open procedure & 1 & 1 & 5 & 6 \\
\hline Reduction by closed procedure & 3 & 1 & 4 & 1 \\
\hline External fixator & 0 & 0 & 2 & 4 \\
\hline \multicolumn{5}{|l|}{ Additional injuries: } \\
\hline Extra-articular hindfoot fractures & 1 & 1 & 3 & 1 \\
\hline Intra-articular hindfoot fractures & 0 & 0 & 3 & 3 \\
\hline Craniocerebral injury II-III & 0 & 0 & 2 & 0 \\
\hline Skin trauma III & 0 & 0 & 2 & 3 \\
\hline
\end{tabular}

Patients were divided into four groups according to the Zwipp score. For each group, mean values of the subjective outcome, the objective clinical outcome, and the radiological findings are shown. Furthermore, the frequency of different types of dislocations, performed treatment, and additional injuries is listed. 
In this study, the long-term outcome was analyzed and the patients were divided into four groups according to their Zwipp score. We aimed to assess the correlation between the clinical outcome and the extent of arthritis and bony hindfoot deformation. A further objective was to relate additional parameters, such as type of dislocation, time until reduction, mode of treatment, and additional injuries to the Zwipp score.

In the excellent and poor patient group, a high correlation between the clinical and radiological findings was observed. However, it is noteworthy that in the good and moderate patient group the CT sores were still relatively high, which was in contrast to the mediocre clinical outcomes. Additional injuries were found to substantially affect the clinical outcome and/or the radiological changes, including severe skin trauma (grade III, Tscherne and Oestern), associated talus and ankle fractures, infection, and traumatic brain injury. Furthermore, we identified factors that appear to have a greater impact on the long-term clinical outcomes than on radiological changes, like severe skin trauma. Interestingly, patients with traumatic brain injuries without associated local fractures showed a poor clinical but a good radiological outcome. Ossifications, however, could not be detected with the computer tomography. Outcome predictors for poor clinical function and radiological findings are associated complex fractures of the hindfoot and infection.

From our results, poor long-term outcomes can also be expected after open dislocations, which is consistent with the findings of a previous report [14]. In contrast, the mode of dislocation (medial or lateral) does not appear to be predictive for long-term complications [13]. With regard to the treatment, reduction by open procedure was often associated with unfavorable long-term results. For closed procedures, however, both good and poor outcomes were observed. The cases exhibiting a poor outcome were all associated with additional injuries. Among all patients, there was only one case in which the medial closed dislocation remained more than 24 hours, that is, clearly exceeding the usual time till reduction of $<6$ hours. Although the patient had no additional injuries, the clinical and radiological results were poor, which confirms the importance of the general recommendation for a fast reduction of dislocations.

Based on our results, it is recommended that, in selected cases, a secondary arthrodesis of the talo-calcanear joint may be an option in order to reduce patient pain and poor clinical function. Subtalar dislocations with associated fractures of the talus and the calcaneus are related to clear signs of osteoarthritis and poor clinical function, a finding that is consistent with previous research [20]. Those patients should be followed up shortly after the acute treatment and an early secondary arthrodesis should be discussed. According to Lasanianos et al., early mobilization should be discussed for patients with isolated medial subtalar dislocations [12]. Furthermore, in cases of subtalar dislocations associated with traumatic brain injury, but without fractures of the hindfoot, residual instability or treatment with an external fixator, and functional rehabilitation (passive motion by a physiotherapist) should be initiated early to combat poor clinical function. With respect to the study limitations, subtalar dislocations are rare injuries, and this study includes only a small number of patients who have been treated in the same hospital. However, according to a meta-analysis of literature pertaining to subtalar dislocations, only 7 of 76 articles reported a higher number of patients than in our study. Nevertheless, the low number of patients in our subgroups must be taken into consideration when interpreting the results of this study.

\section{Conclusion}

Subtalar dislocations are rare, severe injuries, which can lead to long-term complications including poor functional outcomes and arthritis of the ankle joint. In cases where there are additional injuries, especially the presence of traumatic brain injuries, early functional rehabilitation should be considered on a case-by-case basis to ensure a positive clinical outcome.

\section{Acknowledgements}

The authors thank Richard Wagner from our department for his professional advice and help in every part of realizing this study and Torsten Blunk for critically reviewing the manuscript. The first author would like to thank the IZKF (Interdisciplinary Center for Clinical Research), University Clinics of Wuerzburg, for the financial support of her research studies.

\section{Competing Interests Statement}

All authors have no conflict of interest. The study complies with the current laws of Germany. 


\section{References}

[1] Schontag, H., et al. (1984) Late Results in the Treatment of Subtalar Dislocations. Zentralbl Chir, 109, 1436-1440.

[2] DeLee, J.C. and Curtis, R. (1982) Subtalar Dislocation of the Foot. Journal of Bone and Joint Surgery, 64, $433-437$.

[3] Kınık, H., Oktay, O., Arıkan, M. and Mergen, E. (1999) Medial Subtalar Dislocation. International Orthopaedics, 23, 366-367. http://dx.doi.org/10.1007/s002640050396

[4] Freund, K.G. (1989) Subtalar Dislocations: A Review of the Literature. The Journal of Foot Surgery, 28, 429-432.

[5] Perugia, D., et al. (2002) Conservative Treatment of Subtalar Dislocations. International Orthopaedics, 26, 56-60. http://dx.doi.org/10.1007/s002640100296

[6] Marcinko, D.E. and Zenker, C.C. (1991) Peritalar Dislocation without Fracture. The Journal of Foot Surgery, 30, 489-493.

[7] Zimmer, T.J. and Johnson, K.A. (1989) Subtalar Dislocations. Clinical Orthopaedics and Related Research, 238, 190-194. http://dx.doi.org/10.1097/00003086-198901000-00027

[8] Heppenstall, R.B., Farahvar, H., Balderston, R. and Lotke, P. (1980) Evaluation and Management of Subtalar Dislocations. Journal of Trauma, 20, 494-497. http://dx.doi.org/10.1097/00005373-198006000-00011

[9] Marsh, J.L.; Saltzman, C.L., Iverson, M. and Shapiro, D.S. (1995) Major Open Injuries of the Talus. Journal of Orthopaedic Trauma, 9, 371-376. http://dx.doi.org/10.1097/00005131-199505000-00002

[10] Merchan, E.C. (1992) Subtalar Dislocations: Long-Term Follow-Up of 39 Cases. Injury, 23, 97-100. http://dx.doi.org/10.1016/0020-1383(92)90041-P

[11] Bohay, D.R. and Manoli II, A. (1995) Subtalar Joint Dislocations. Foot \& Ankle International, 16, 803-808. http://dx.doi.org/10.1177/107110079501601212

[12] Lasanianos, N.G., Lyras, D.N., Mouzopoulos, G., Tsutseos, N. and Garnavos, C. (2011) Early Mobilization after Uncomplicated Medial Subtalar Dislocation Provides Successful Functional Results. Journal of Orthopaedics and Traumatology, 12, 37-43. http://dx.doi.org/10.1007/s10195-011-0126-2

[13] Jungbluth, P., Wild, M., Hakimi, M., Gehrmann, S., Djurisic, M., Windolf, J., et al. (2010) Isolated Subtalar Dislocation. Journal of Bone and Joint Surgery (American), 92, 890-894.

http://dx.doi.org/10.2106/JBJS.I.00490

[14] Milenkovic, S., Mitkovic, M. and Bumbasirevic, M. (2006) External Fixation of Open Subtalar Dislocation. Injury, 37, 909-913. http://dx.doi.org/10.1016/j.injury.2006.02.051

[15] De Palma, L., Santucci, A., Marinelli, M., Borgogno, E. and Catalani, A. (2008) Clinical Outcome of Closed Isolated Subtalar Dislocations. Archives of Orthopaedic and Trauma Surgery, 128, 593-598. http://dx.doi.org/10.1007/s00402-007-0459-8

[16] Garofalo, R., Moretti, B., Ortolano, V., Cariola, P., Solarino, G., Wettstein, M. and Mouhsine, E. (2004) Peritalar Dislocations: A Retrospective Study of 18 Cases. Journal of Foot and Ankle Surgery, 43, 166-172. http://dx.doi.org/10.1053/j.jfas.2004.03.008

[17] Wagner, R., Blattert, T.R. and Weckbach, A. (2004) Talar Dislocations. Injury, 35, 36-45. http://dx.doi.org/10.1016/j.injury.2004.07.010

[18] Tucker, D.J., Burian, G. and Boylan, J.P. (1998) Lateral Subtalar Dislocation: Review of the Literature and Case Presentation. Journal of Foot and Ankle Surgery, 37, 239-247. http://dx.doi.org/10.1016/S1067-2516(98)80118-X

[19] Hoexum, F. and Heetveld, M.J. (2014) Subtalar Dislocation: Two Cases Requiring Surgery and a Literature Review of the Last 25 Years. Archives of Orthopaedic and Trauma Surgery, 134, 1237-1249. http://dx.doi.org/10.1007/s00402-014-2040-6

[20] Simon, L.C., Schulz, A.P., Faschingbauer, M., Morlock, M. and Jürgens, C. (2008) "Basketball Foot”-Long-Time Prognosis after Peritalar Dislocation. Sportverletzung Sportschaden, 22, 31-37.

http://dx.doi.org/10.1055/s-2008-1027208 